\title{
Stereotactic Laser Ablation (SLA) Followed by Consolidation Stereotactic Radiosurgery (cSRS) as Treatment for Brain Metastasis that Recurred Locally After Initial Radiosurgery (BMRS): A Multi-Institutional Experience
}

\section{Isabela Peña Pino}

University of Minnesota Medical Center Fairview: University of Minnesota Health Jun Ma

University of Minnesota Medical Center Fairview: University of Minnesota Health

\section{Yusuke Hori}

Cleveland Clinic Lerner College of Medicine of CWRU: Cleveland Clinic Lerner College of Medicine of Case Western Reserve University

Elena Fomchenko

Yale University School of Medicine

\section{Kathryn Dusenbery}

University of Minnesota Medical Center Fairview: University of Minnesota Health

\section{Margaret Reynolds}

University of Minnesota Medical Center Fairview: University of Minnesota Health

\section{Christopher Wilke}

University of Minnesota Medical Center Fairview: University of Minnesota Health

Jianling Yuan

University of Minnesota Medical Center Fairview: University of Minnesota Health

\section{Ethan Srinivasan}

Duke University Medical Center: Duke University Hospital

\section{Matthew Grabowski}

Duke University Medical Center: Duke University Hospital

\section{Peter Fecci}

Duke University Medical Center: Duke University Hospital

\section{Evidio Domingo-Musibay}

University of Minnesota Medical Center Fairview: University of Minnesota Health

\section{Naomi Fujioka}

University of Minnesota Medical Center Fairview: University of Minnesota Health

\section{Gene H. Barnett}

Cleveland Clinic Lerner College of Medicine of CWRU: Cleveland Clinic Lerner College of Medicine of Case Western Reserve University 


\section{Veronica Chang}

Yale University School of Medicine

Alireza M. Mohammadi

Cleveland Clinic Lerner College of Medicine of CWRU: Cleveland Clinic Lerner College of Medicine of Case Western Reserve University

Clark C Chen ( $\square$ ccchen@umn.edu )

University of Minnesota Health https://orcid.org/0000-0001-6258-9277

\section{Research Article}

Keywords: Brain metastasis (BM), Stereotactic laser ablation (SLA), laser interstitial thermal therapy (LITT), stereotactic radiosurgery (SRS)

Posted Date: August 11th, 2021

DOl: https://doi.org/10.21203/rs.3.rs-794328/v1

License: (1) This work is licensed under a Creative Commons Attribution 4.0 International License. Read Full License 


\section{Abstract}

Introduction: The optimal treatment paradigm for brain metastasis that recurs locally after initial radiosurgery (BMRS) remains an area of active investigation. Here, we report outcomes for patients with BMRS treated with stereotactic laser ablation (SLA, also known as laser interstitial thermal therapy, LITT)followed by consolidation radiosurgery (cSRS).

Methods: Clinical outcome of 20 patients with 21 histologically confirmed BMRS treated with SLA followed by consolidation SRS and $>6$ months follow-up were collected retrospectively across three participating institutions.

Results: Consolidation SRS ( 5 Gy $\times 5$ or 6 Gy x 5) wascarried out $16-73$ days (median of 26 days) post-SLA of BMRS. There were no new neurological deficits after SLA/cSRS. While $3 / 21(14.3 \%)$ patients suffered temporary Karnofsky Performance Score (KPS) decline after SLA, no KPS declines was observed after cSRS. There were no 30-day mortalities or wound complications. Two patients required re-admission within 30 days of cSRS (severe headache that resolved with steroid therapy $(n=1)$ and new onset seizure $(n=1))$. With a median follow-up of 228 days (range: 178-1367 days), the local control rate at 6 and 12 months $\left(\mathrm{LC}_{6}, \mathrm{LC}_{12}\right)$ was $100 \%$. All showed diminished FLAIR volume surrounding the SLA/cSRS treated BMRS at the six-month follow-up; none of the patients required steroid for symptoms attributable to these BMRS. These results compare favorably to the available literature for repeat SRS or SLA-only treatment of BMRS.

Conclusions: This multi-institutional experience supports further investigations of SLA/cSRS as a treatment strategyfor BMRS.

\section{Introduction}

In independent randomized clinical trials, $\sim 30 \%$ of BM recur locally after radiosurgery twelve months after treatment [1]. The local recurrence rate is higher for BM intrinsically resistant to radiation [2, 3]or those with upregulated DNA repair capacity [4-6]. There is currently no consensus on the optimal treatment strategy for brain metastasis that recur after radiosurgery (BMRS). Repeat radiosurgery to BMRS is often employed in this context. However, such treatments are associated with an increased risk for neurologic decline secondary to adverse radiation effects, including radiation necrosis[7, 8]. These repeat radiation-associated deleterious effects can compromise the patient's quality of life, delay systemic therapy, or necessitate surgical interventions [8, 9]. Moreover, up to $30 \%$ of repeat radiosurgery fail to achieve local control of BMRS [10].

Stereotactic laser ablation (SLA, also known as laser interstitial thermal therapy (LITT)) refers to a procedure whereby a fiberoptic laser probe is stereotactically inserted into a target abnormality in the brain or spine [11]. Activation of the probe induces hyperthermia within the target tissue, causing coagulative necrosis [11]. While SLA has been employed as a treatment for newly diagnosed BM, the clinical outcomes have been disappointing, with 30-50\% local failure within twelve months of treatment [12-15]. Despite these results, there are reasons to consider SLA in combination with repeat radiosurgery as a treatment strategy for BMRS. First, there is sound laboratory science [16], pre-clinical [17], and clinical data [18-20] suggesting synergy in the antitumoral effects of hyperthermia and radiation. This synergy warrant consideration given the radiation resistant nature of BMRS. Second, SLA is a highly effective treatment for radiosurgery associated adverse radiation 
effects $[14,21,22]$. The available literature suggests that SLA inactivates immune cells and/or denatures inflammatory cytokines that mediate radiation-induced injuries [23]. Thus, the addition of SLA to repeat SRS can potentially mitigate risk of subsequent adverse radiation effects.

In this context, we previously reported results from four BMRS patients treated with SLA followed by cSRS in 2016 [13]. Here, we report a larger multi-institutional experience examining the efficacy and safety profile of this treatment strategy for patients with $>6$ months follow-up.

\section{Methods}

\section{Patient population and data collection}

This study was a multi-institutional, retrospective case series of patients who underwent SLA/cSRS between 2012 and 2020 for needle biopsy-confirmed brain metastasis recurrence after initial SRS treatment. Each of the participating institutions holds an approved IRB for this retrospective review. To characterize longer-term effects of SLA/cSRS, we included only patients who had 6 months follow-up data. Demographic and clinical data collected from the electronic record system included: sex, age at BMRS presentation, primary tumor subtype, tumor location, tumor volume, prior whole brain radiation therapy (WBRT), 30-day readmission, and SLA/cSRS associated morbidity (including radiation induced adverse effects). Baseline and post-

SLA/cSRSKarnofsky Performance Status (KPS) and steroid use were collected for all patients at time of BMRS diagnosis, one day post-SLA, one monthpost-cSRS, and at subsequent follow-up visits. As per standard of care, patients were seen by the neurosurgeon $\sim 2$ weeks after SLA. Patients were seen by the radiation oncologist one month after cSRS and every 2-3 months thereafter. Follow-up duration was calculated from the time of cSRS.Overall Survival was calculated from the time of initial cancer diagnosis.

\section{MRI analysis}

All patients underwent surveillance MRI every 2-3 months unless imaging or clinical findings warranted shorter interval follow-up. MRls from each site are reviewed by two independent assessors. MRIs were obtained before and after treatment and imported into the Brainlab (BrainlabAG,Munich, Germany)iPlan Cranial 3.0 software or 3D Slicer (www.slicer.org) or image J (https://imagej.nih.gov/ij/) to determine maximal diameter and volume. The volume of contrast enhancing (CE) and FLAIR volumes surrounding the SLA/cSRS treated lesion were recorded immediately post-SLA and for each imaging follow-up. Local control (LC) was defined using modified RANO-BM criteria [24], as described below. Since the SLA ablated region often show expansion of contrast enhancement immediately post-procedure that resolves in the subsequent one to three months [25], we define local failure as persistent increase of $>20 \%$ in the size of contrast enhancement (based on RANO-BM) relative to the time of BMRS presentation, on two sequential MRIs that were greater than three months apart (similar to immunotherapy RANO [26]). Lesions demonstrating such local failure were surgically excised for definitive tissue diagnosis.

\section{SLA and consolidation SRS}

BMRS patients considered for SLA/CSRS were reviewed at a multi-disciplinary board consisting of representatives from neuro-oncology, neurosurgery, radiation oncology, neuroradiology, and neuropathology. 
Informed consents were obtained by neurosurgery and radiation oncology. The SLA procedures were performed as previously described [13]. For lesions treated with the NeuroBlate system (Monteris Medical Inc., Plymouth, MN, USA), extent of ablation was assessed based on CE region covered by blue contour line (equivalent to of tissue heating to $43^{\circ} \mathrm{C}$ for $>10$ minutes) [27]. For lesions treated with the Visualase system (Medtronics Inc., Minneapolis, $\mathrm{MN}$ ), extent of ablation was assessed based on $\mathrm{CE}$ region covered by orange pixel (equivalent to tissue injury achieved at $60^{\circ} \mathrm{C}$ ).

Consolidation SRS was carried out approximately one to two months after the SLA and delivered through five consecutive fractions of 5 Gy or 6 Gy. SRS treatment was performed using either the Leksell Gamma Knife Icon (Elekta Instrument AB, Stockholm, Sweden) or the TrueBeam linear accelerator (Varian Medical Systems, Palo Alto, California). The planned target volume (PTV) was defined by the entirety of the ablated lesion, including, regions of contrast enhancement (Figure 1). For Icon-treated patients, PTV was prescribed to the $50 \%$ isodose. For the TrueBeam treated patients, PTV was prescribed to the $90 \%$ isodose.

\section{Literature search and statistical analysis}

The literature search was performed in June 2021, reviewed by the first author (IP) and senior author (CCC). For assessment of the published literature for SLA as treatment for BMRS, we repeated a search strategy that we had previously implemented in 2018 [13]. In this search, we identified two publications bearing information on the local control of BMRS after SLA. For assessment of the published literature for hypo-fractionated repeat SRS as treatment for BMRS, we repeated a published strategy that was implemented in 2019 by Loi et al [28]. We identified four publications bearing pertinent local control and adverse radiation effect information following repeat, hypo-fractionated radiosurgery of BMRS.

GraphPad Prism Version 9 was used for all statistical analyses (GraphPad Software, San Diego, California). Categorical and continuous data are described as frequency (percentage) and median (interquartile range), respectively. Fisher's exact test and the Mann-Whitney U-test were used for the univariate analyses of categorical and continuous data, respectively.

\section{Results}

\section{Study population}

The study cohort consists of six male and thirteen female patients. The median age of the cohort was 64 years old (range: 34-74). The histology of the BMs treated included: lung $(n=6)$, breast $(n=5)$, gastrointestinal $(n=3)$, melanoma $(n=2)$, laryngeal $(n=1)$, urothelial $(n=1)$, ovarian $(n=1)$, and undifferentiated $(n=1)$. The medianCE tumor volume at the time of BMRS presentation was $5.6 \mathrm{~cm}^{3}$ (range: $3.1-34.2 \mathrm{~cm}^{3}$ ). All lesions were deemed difficult to access surgically by the treating neurosurgeon and underwent stereotactic needle biopsies, with pathology confirming BMRS. All lesions received 18-21 Gy of single fraction SRS at the time of initial diagnosis. The locations of the lesions as well as other demographic and tumor characteristics are shown in Table 1. Except for patient 11, who was treated with WBRT two years prior to the initial SRS, none of the patients received prior WBRT. Patient 4 underwent two separate SLA/cSRS for lesions located in the frontal and parietal lobe, identified approximately six months apart. All other patients underwent a single SLA/cSRS. All 
patients were treated with corticosteroid following the BMRS diagnosis and prior to SLA. All patients underwent subsequent systemic therapy.

\section{SLA/cSRS and post-operative course}

The volume of BMRS CE ablated was as defined in Methods. Seventy-three to $100 \%$ of the BMRS CE volume was ablated at the time of SLA (Table 2). There were no new neurologic deficits immediately post-SLA or at the one-month follow-up. Consistent with previously published SLA series [14, 29], the KPS of $80 \%(16 / 20)$ of the patients remain unchanged after SLA. Three of 20 (15\%) suffered a temporary decline in KPS post-SLA, while $1 / 20(5 \%)$ showed an improvement in KPS. All patients were discharged home within two days of SLA. There was no operative morbidity or mortality attributable to the SLA.

Consolidation SRSwas performed one to two months after SLA and was performed as an out-patient procedure. The median time from SLA to cSRS was 26 days (range 16-73 days). The median BMRS CE volume for this series was $5.6 \mathrm{~cm}^{3}$. cSRS was targeted the post-ablative region, including the rim of contrast enhancement (Figure 1). Eighteen of 20 (90\%) of the patients were treated with a 5 Gy x 5 consecutive day regimen, and 2 patients were treated with a 6 Gy x 5 consecutive day regimen. By the time of cSRS, the three patients who suffered a KPS decline after SLA had recovered to their baseline KPS. All KPS remained stable at one-month post-cSRS.

There were two 30-day post-SLA readmissions. Patient 7 presented with an incapacitating headache in the context of corticosteroid dose. The headache resolved after a temporary re-escalation of corticosteroid treatment. Patient 11 presented with a new-onset seizure and was placed on levetiracetam and a short course of corticosteroid. Of note, patient 11 is the only patient in this series who received WBRT prior to initial SRS. Head CT's for both patients were without evidence of tumor hemorrhage but showed increased per-BMRS hypodensity. Both patients were discharged by hospital day 2. Of note, both admitted patients harbored BMRS that is larger than the median volume of the tumor treated in this series. The median BMRS CE volume for this series was $5.6 \mathrm{~cm}^{3}$; the BMRS CE volumes for patients 7 and 11 were $35 \mathrm{~cm}^{3}$ and $25.7 \mathrm{~cm}^{3}$, respectively.

\section{Imaging and clinical follow-up}

Follow-up duration and local control was calculated from the time of cSRS. The median follow-up for this cohort is 228 days (range: 183-1367 days). There were two treatment failures that occurred 1367 and 1161 days after SLA/cSRS (patients 13 and 14, Figure 2A). Overall survival was calculated from the time of the cancer diagnosis. The median survival for this study cohort was 378 days (Figure 2B).Six months follow-up was available for all patients (Table 3). At six months, the CE volumes for all SLA/cSRS treated lesions were decreased relative to the time of SLA. Similarly, the peri-CE FLAIR volumes for all treated lesionsdecreased relative to the initial BMRS presentation. Six months after SLA/cSRS, five out of 20 (25\%) of the patients continued to be treated with corticosteroid. Based on both clinical documentation and surveillance MR imaging, the corticosteroid was used to treat symptoms related to other BM and unrelated to the SLA/cSRS treated BMRS,

Twelve-month follow-up was available for five patients. Local control at twelve months ( $\left.\mathrm{LC}_{12}\right)$ was $100 \%$, and the peri-CE FLAIR volumes remained decreased for the treated lesions relative to the time of SLA. None of the 
patients required corticosteroid treatment. Two illustrative cases are shown in Figures 3and 4. Figure 3shows the evolution of CE and FLAIR after SLA/cSRS in the longest surviving patient (patient 8) whose BMRS remain controlled 19 months after SLA/cSRS. Figure 4 shows the evolution of CE and FLAIR after SLA/cSRS in a patient (patient 14) whose BMRS recurred 1161 days( 39 months) after SLA/cSRS.

\section{Literature of efficacy of repeat radiosurgery and stereotactic laser ablation (SLA)}

We wishto compare our case series to the published literature for salvage monotherapy using either SLA or repeat SRS for BMRS. The literature search for SLA treated BMRS (see Methods for the search algorithm) yielded one study that reported a local control of $61 \%$ at 4.6 months [13]. Broadening our search to include case series that include a mixture of pathology confirmed BMRS, newly diagnosed BMRS, and radiation necrosis, we identified two additional studies. The study by Ahluwalia et al. consisted of 22 pathologyconfirmed BMRS and 22 radiation necrosis. Only three-month local control was reported $\left(\mathrm{LC}_{3}\right)$, and the reported $\mathrm{LC}_{3}$ was $37 \%$ [14]. The second study by Bastos et al. consisted of 46 BMRS and 5 newly diagnosed BM. The reported $L_{12}$ was $69.6 \%$ for this series [15] (Table 4A).

Since our cSRSused a hypo-fractionated regimen, we focused our literature search to identify articles that studied the efficacy and safety of hypo-fractionated repeat SRS as a treatment for BMRS. We identified four such articles (Table 4B) [10, 30-32]. The reported local control at 12 months ranged 70-83\%, with 13-19\% of the patients suffering from symptomatic adverse radiation effects after repeat radiation.

The $\mathrm{LC}_{12}$ of $100 \%$ and $0 \%$ adverse radiation effects at 6 and 12 months after SLA/cSRS treatment of BMRS in our series compares favorably to these reported results.

\section{Discussion}

Our literature search suggests that the treatment of BMRS remains an understudied area in neuro-oncology. The overall poor survival of BM patients [33] likely contributes to this limited literature. Many BM patients die before developing recurrence. However, as newer classes of systemic therapies emerge and improve cancer survival, there will be a greater need for meaningful therapeutic interventions in the treatment of BMRS [34]. Here, we retrospectively analyzed a multi-institutional experience that employed SLA/cSRS as a treatment strategy for BMRS. This study builds on and expands on the clinical outcome of the four patients reported in our 2016 study [13]. By expanding the number of patients treated as well as lengthening the follow-up duration (to > 6 months), we provide data to support the safety profile and durable efficacy of SLA/cSRS as treatment for BMRS.

Our current study suggests that the procedural safety profile of SLA/cSRS is comparable to that reported for SLA alone $[13,14,35]$. Because of the limited BMRS literature, we were unable to perform a meaningful, quantitative comparison between the results of this study to the available literature. Qualitatively, the $\mathrm{LC}_{6}$ and $\mathrm{LC}_{12}$ of $100 \%$ after SLA/cSRS for tissue confirmed BMRS in our series compares favorably to the reported $\mathrm{LC}_{12}$ of $69.6 \%$ [15] for SLA alone. Of note, this SLA study included a mixed population of BMRS and newly diagnosed BM, with the latter presumably more radiation sensitive than the former. The local control of BMRS by SLA/cSRS also compares favorably to the 70-83\% reported for repeat hypo-fractionated SRS. These results should be interpreted with the following consideration. While it is unlikely that any practitioner would purposely 
treat non-recurrent BM with repeat radiosurgery, the imaging methods available for assessing recurrent BM versus adverse radiation effects following radiosurgery remain imperfect [36]. As such, it is possible that a subset of the lesions thought to be BMRS on imaging may represent post-radiosurgery radiation effects. If so, the published study of BMRS without tissue diagnosis reports local control rates that may be artificially inflated [37]. In this context, it is notable that all BMRS in this series were confirmed by tissue.

Another advantage of the SLA/cSRS treatment paradigm is highlighted by the absence of adverse radiation effects in our series compared to the13-19\% reported withrepeat radiosurgery of BMRS. The neurologic sequelae of these adverse effects compromise the patient's quality of life, requiring corticosteroid therapy, surgical therapy, or both. Since innate anti-tumoral responses play a key role in the efficacy of nearly all anticancer therapies, immune suppression secondary to corticosteroid administration may compromise the efficacy of systemic therapies [38]. Moreover, the advent of immunotherapy and targeted therapy has increased the risk for SRS-associated adverse radiation effects $[39,40]$. As many as one in five melanoma BM patients treated with nivolumab and ipilimumab suffers adverse radiation effects after SRS, requiring cessation of systemic therapy [40]. Minimizing the risk of such cessation has the potential to improve therapeutic efficacy of systemic therapy. In this context, the absence of adverse radiation effects in our SLA/cSRS series despite repeat radiosurgery to the same lesion suggests that SLA may minimize the risk of adverse radiation effects,rendering the treatment strategy attractive.

The imaging findings after SLA-cSRS are complex and worth noting. MRIs performed immediately post-SLA typically show an expansion in CE volume, accompanied by an enlargement of the surrounding FLAIR [27, 35]. While many SLA-treated lesions show egg-shell contrast enhancement (Figures 3 and 4), others exhibit more heterogeneous enhancement patterns $[12,13,41]$. These SLA-associated MR changes typically resolve one to three months post-SLA $[12,13,35,41]$. As such, assessment of MR at any single time point after SLA, such as that described by RANO-BM [42], is inadequate for assessing tumor control. In this context, we modified the RANO-BM criteria to include the sequential surveillance MRIs, similar to that proposed for immunotherapy RANO (iRANO) [26]. We considered local failure only if the $\geq 20 \%$ increase in CE volume remained persistent on MRIsscanned three months apart. The pilot data presented in our series support the utility of this proposed modification. Of the two cases (patient 13 and 14) that showed tumor progression at later time points based on the above proposed criteria, both underwent surgical resection, with specimens confirming tumor progression.

An important consideration in patient selection for SLA involves the volume of the BMRS. Post-SLA edema is a well-documented phenomenon that can lead to morbidities and mortalities [13,35]. In this context, the larger lesions treated in this series involved patients with either cerebral atrophy or who had undergone previous surgical resections. The atrophy or resection reduce cerebral volume to increase capacity for tolerating postSLA edema. Despite the cautions exercised in patient selection, two of the patients (patient 7 and 11) with larger BMRS suffered 30-day readmission after SLA/cSRS for symptoms attributable to treated BMRS. Patient 11 's prior history of WBRT further suggest that the cumulative radiation dose may influence cerebral compliance and tolerance of post-SLA edema. As such, judicious consideration in patient selection and close follow-up of SLA/cSRS treated patients is warranted, especially for patients with larger tumor volumes or patients who had undergone multiple rounds of prior RT/SRS. 
As a retrospective analysis, our conclusions are subject to the influences of all forms of biases inherent within this study design [27]. Moreover, all BMRS presented in this series underwent SLA that achieved $>70 \%$ ablation of the contrast enhancing tumor volume. As such, the results reported here may not be applicable if such extent of ablation was not achieved. Ultimately, a randomized control trial (RCT) will be needed to validate the results reported here. If the pilot safety and efficacy profiles reported here are recapitulated in an RCT, the results can contribute to a new standard of care for BMRS patients. While this case series focused exclusively on patients with recurrent $\mathrm{BM}$, the approach should be applicable to other clinical contexts, including the treatment of BM with a high probability of SRS failure, such as larger volume melanoma BM in the upfront setting.

\section{Conclusion}

This multi-institutional experience provides pilot safety and efficacy data for hypo-fractionated SRS within two months of SLA treatment of BMRS and support continued investigation of this treatment strategy. The results described here informs the design of future randomized clinical trials.

\section{Declarations}

Funding: No funding was provided for this study.

Conflicts of interest: The authors do not have any conflicts of interest to report

Availability of data: Data will be made available upon request as deemed appropriate by the corresponding author

Code availability: N/A

Authors' contributions: JM, YH, EF, KD, MR, CW, JY, ES, MG, PF, EDM, NF, GHB, VC and AM collected data. IPP drafted the manuscript. CCC conceived the study, performed procedures reported, drafted the manuscript. All authors edited the final draft of the manuscript and provided critical review.

Ethics approval: This study was approved by the local IRB at each participating institution.

Consent to participate: Consent for data collection was waived as this was a retrospective study.

Consent for publication: N/A, no private health information is published in this manuscript.

\section{References}

1. Roberge D, Brown PD, Whitton A, et al (2018) The Future Is Now-Prospective Study of Radiosurgery for More Than 4 Brain Metastases to Start in 2018! Front Oncol 8:380

2. Douglas JG, Margolin K (2002) The treatment of brain metastases from malignant melanoma. Semin Oncol 29:518-524

3. Christ SM, Mahadevan A, Floyd SR, et al (2015) Stereotactic radiosurgery for brain metastases from malignant melanoma. Surg Neurol Int 6:S355-65 
4. Kao WH, Riker Al, Kushwaha DS, et al (2011) Upregulation of Fanconi anemia DNA repair genes in melanoma compared with non-melanoma skin cancer. J Invest Dermatol 131:2139-2142

5. Zohrabian VM, Nandu H, Gulati N, et al (2007) Gene expression profiling of metastatic brain cancer. Oncol Rep 18:321-328

6. Lamb R, Fiorillo M, Chadwick A, et al (2015) Doxycycline down-regulates DNA-PK and radiosensitizes tumor initiating cells: Implications for more effective radiation therapy. Oncotarget 6:14005-14025

7. Koffer P, Chan J, Rava P, et al (2017) Repeat Stereotactic Radiosurgery for Locally Recurrent Brain Metastases. World Neurosurg 104:589-593

8. Iorio-Morin C, Mercure-Cyr R, Figueiredo G, et al (2019) Repeat stereotactic radiosurgery for the management of locally recurrent brain metastases. J Neurooncol 145:551-559

9. Koiso T, Yamamoto M, Kawabe T, et al (2016) Follow-up results of brain metastasis patients undergoing repeat Gamma Knife radiosurgery. J Neurosurg 125:2-10

10. Minniti G, Scaringi C, Paolini S, et al (2016) Repeated stereotactic radiosurgery for patients with progressive brain metastases. J Neurooncol 126:91-97

11. Franzini A, Moosa S, Servello D, et al (2019) Ablative brain surgery: an overview. Int J Hyperthermia 36:6480

12. Carpentier A, McNichols RJ, Stafford RJ, et al (2011) Laser thermal therapy: real-time MRI-guided and computer-controlled procedures for metastatic brain tumors. Lasers Surg Med 43:943-950

13. Ali MA, Carroll KT, Rennert RC, et al (2016) Stereotactic laser ablation as treatment for brain metastases that recur after stereotactic radiosurgery: a multiinstitutional experience. Neurosurg Focus 41:E11

14. Ahluwalia M, Barnett GH, Deng D, et al (2018) Laser ablation after stereotactic radiosurgery: a multicenter prospective study in patients with metastatic brain tumors and radiation necrosis. J Neurosurg 130:804811

15. Bastos DC de A, Rao G, Oliva ICG, et al (2020) Predictors of Local Control of Brain Metastasis Treated With Laser Interstitial Thermal Therapy. Neurosurgery 87:112-122

16. Rezaie P, Khoei S, Khoee S, et al (2018) Evaluation of combined effect of hyperthermia and ionizing radiation on cytotoxic damages induced by IUdR-loaded PCL-PEG-coated magnetic nanoparticles in spheroid culture of U87MG glioblastoma cell line. Int J Radiat Biol 94:1027-1037

17. Overgaard J (1989) The current and potential role of hyperthermia in radiotherapy. Int J Radiat Oncol Biol Phys 16:535-549

18. Dewey WC (2009) Arrhenius relationships from the molecule and cell to the clinic. Int J Hyperthermia 25:3-20

19. Datta NR, Ordóñez SG, Gaipl US, et al (2015) Local hyperthermia combined with radiotherapy and-/or chemotherapy: recent advances and promises for the future. Cancer Treat Rev 41:742-753

20. Grauer O, Jaber M, Hess K, et al (2019) Combined intracavitary thermotherapy with iron oxide nanoparticles and radiotherapy as local treatment modality in recurrent glioblastoma patients. J Neurooncol 141:83-94

21. Hong CS, Beckta JM, Kundishora AJ, et al (2020) Laser interstitial thermal therapy for treatment of cerebral radiation necrosis. Int $\mathrm{J}$ Hyperthermia 37:68-76

Page $10 / 20$ 
22. Chen C, Lee I, Tatsui C, et al (2021) Laser interstitial thermotherapy (LITT) for the treatment of tumors of the brain and spine: a brief review. J Neurooncol 151:429-442

23. Munier S, Ginalis EE, Patel NV, et al (2020) Radiation Necrosis in Intracranial Lesions. Cureus12:e7603

24. Lin NU, Lee EQ, Aoyama H, et al (2015) Response assessment criteria for brain metastases: proposal from the RANO group. Lancet Oncol 16:e270-8

25. Beechar VB, Prabhu SS, Bastos D, et al (2018) Volumetric response of progressing post-SRS lesions treated with laser interstitial thermal therapy. J Neurooncol 137:57-65

26. Wen PY, Chang SM, Van den Bent MJ, et al (2017) Response Assessment in Neuro-Oncology Clinical Trials. $\mathrm{J}$ Clin Oncol 35:2439-2449

27. Sloan AE, Ahluwalia MS, Valerio-Pascua J, et al (2013) Results of the NeuroBlate System first-in-humans Phase I clinical trial for recurrent glioblastoma: clinical article. J Neurosurg 118:1202-1219

28. Loi M, Caini S, Scoccianti S, et al (2020) Stereotactic reirradiation for local failure of brain metastases following previous radiosurgery: Systematic review and meta-analysis. Crit Rev Oncol Hematol 153:103043

29. Kim AH, Tatter S, Rao G, et al (2020) Laser Ablation of Abnormal Neurological Tissue Using Robotic NeuroBlate System (LAANTERN): 12-Month Outcomes and Quality of Life After Brain Tumor Ablation. Neurosurgery 87:E338-E346

30. Holt DE, Gill BS, Clump DA, et al (2015) Tumor bed radiosurgery following resection and prior stereotactic radiosurgery for locally persistent brain metastasis. Front Oncol 5:84

31. Rana N, Pendyala P, Cleary RK, et al (2017) Long-term Outcomes after Salvage Stereotactic Radiosurgery (SRS) following In-Field Failure of Initial SRS for Brain Metastases. Front Oncol 7:279

32. Dincoglan F, Sager O, Demiral S, et al (2019) Fractionated stereotactic radiosurgery for locally recurrent brain metastases after failed stereotactic radiosurgery. Indian J Cancer 56:151-156

33. Niranjan A, Monaco E, Flickinger J, Lunsford LD (2019) Guidelines for Multiple Brain Metastases Radiosurgery. Prog Neurol Surg 34:100-109

34. Achrol AS, Rennert RC, Anders C, et al (2019) Brain metastases. Nat Rev Dis Primers 5:5

35. Alattar AA, Bartek J Jr, Chiang VL, et al (2019) Stereotactic Laser Ablation as Treatment of Brain Metastases Recurring after Stereotactic Radiosurgery: A Systematic Literature Review. World Neurosurg 128:134-142

36. Verma N, Cowperthwaite MC, Burnett MG, Markey MK (2013) Differentiating tumor recurrence from treatment necrosis: a review of neuro-oncologic imaging strategies. Neuro Oncol 15:515-534

37. Alomari A, Rauch PJ, Orsaria M, et al (2014) Radiologic and histologic consequences of radiosurgery for brain tumors. J Neurooncol 117:33-42

38. Dixit KS, Kumthekar PU (2020) Optimal Management of Corticosteroids in Patients with Intracranial Malignancies. Curr Treat Options Oncol 21:77

39. Ramakrishna R, Formenti S (2019) Radiosurgery and Immunotherapy in the Treatment of Brain Metastases. World Neurosurg 130:615-622

40. Kroeze SGC, Fritz C, Hoyer M, et al (2017) Toxicity of concurrent stereotactic radiotherapy and targeted therapy or immunotherapy: A systematic review. Cancer Treat Rev 53:25-37

Page $11 / 20$ 
41. Schwabe B, Kahn T, Harth T, et al (1997) Laser-induced thermal lesions in the human brain: short- and long-term appearance on MRI. J Comput Assist Tomogr 21:818-825

42. Camidge DR, Lee EQ, Lin NU, et al (2018) Clinical trial design for systemic agents in patients with brain metastases from solid tumours: a guideline by the Response Assessment in Neuro-Oncology Brain Metastases working group. Lancet Oncol 19:e20-e32

\section{Tables}

\section{TABLE 1. Patient demographics and lesion characteristics}




\begin{tabular}{|c|c|c|c|c|c|c|c|c|c|}
\hline Patient & Age & Sex & $\begin{array}{l}\text { Pre- } \\
\text { SLA } \\
\text { KPS }\end{array}$ & $\begin{array}{l}\text { Pre-SLA } \\
\text { corticosteroid }\end{array}$ & Primary Tumor & Location & $\begin{array}{l}\text { Lesion } \\
\text { volume } \\
\left(\mathrm{cm}^{3}\right)\end{array}$ & $\begin{array}{l}\text { Prior } \\
\text { WBRT }\end{array}$ & $\begin{array}{l}\text { Prior } \\
\text { SRS } \\
\text { dose } \\
\text { (Gy) }\end{array}$ \\
\hline 1 & 67 & $\mathrm{~F}$ & 90 & $\mathrm{Y}$ & Colon & $\begin{array}{l}\mathrm{L} \\
\text { thalamus }\end{array}$ & 3.4 & $\mathrm{~N}$ & 20 \\
\hline 2 & 58 & $\mathrm{~F}$ & 100 & Y & Melanoma & $\begin{array}{l}\mathrm{L} \\
\text { thalamus }\end{array}$ & 4.5 & $\mathrm{~N}$ & 20 \\
\hline 3 & 54 & $\mathrm{~F}$ & 90 & Y & Breast & $\begin{array}{l}\mathrm{R} \\
\text { thalamus }\end{array}$ & 5.5 & $\mathrm{~N}$ & 20 \\
\hline $4 *$ & 60 & $\mathrm{~F}$ & 80 & Y & Breast & $L$ frontal & 3.2 & $\mathrm{~N}$ & 20 \\
\hline $4^{*}$ & 60 & $\mathrm{~F}$ & 80 & $\mathrm{Y}$ & Breast & L parietal & 4.9 & $\mathrm{~N}$ & 20 \\
\hline 5 & 73 & $M$ & 100 & Y & Lung & $\begin{array}{l}\mathrm{R} \\
\text { temporal }\end{array}$ & 4.2 & $\mathrm{~N}$ & 20 \\
\hline 6 & 69 & $\mathrm{~F}$ & 100 & Y & Ovarian & $\begin{array}{l}\mathrm{L} \\
\text { cerebellar }\end{array}$ & 15.9 & $\mathrm{~N}$ & 18 \\
\hline 7 & 73 & $\mathrm{~F}$ & 70 & Y & Urothelial & $\begin{array}{l}\text { L peri- } \\
\text { atrial }\end{array}$ & 35 & $\mathrm{~N}$ & 18 \\
\hline 8 & 53 & $\mathrm{~F}$ & 100 & $Y$ & Esophageal & $\begin{array}{l}\mathrm{L} \\
\text { temporal }\end{array}$ & 4.5 & $\mathrm{~N}$ & 20 \\
\hline 9 & 34 & $M$ & 70 & $Y$ & Melanoma & $\begin{array}{l}\text { Rperi- } \\
\text { atrial }\end{array}$ & 34.2 & $\mathrm{~N}$ & 18 \\
\hline 10 & 66 & $\mathrm{~F}$ & 80 & $\mathrm{Y}$ & Breast & L parietal & 27.1 & $\mathrm{~N}$ & 18 \\
\hline 11 & 66 & $\mathrm{~F}$ & 60 & Y & Lung & $\begin{array}{l}\mathrm{L} \\
\text { cingulate }\end{array}$ & 25.7 & Y & 18 \\
\hline 12 & 58 & $\mathrm{~F}$ & 90 & Y & SCC larynx & R parietal & 3.6 & $\mathrm{~N}$ & 20 \\
\hline 13 & 74 & $\mathrm{~F}$ & 80 & Y & Lung & L parietal & 14 & $\mathrm{~N}$ & 18 \\
\hline 14 & 64 & $\mathrm{~F}$ & 60 & Y & Breast & L parietal & 5.6 & $\mathrm{~N}$ & 20 \\
\hline 15 & 57 & $M$ & 90 & $\mathrm{Y}$ & Colon & $\begin{array}{l}\mathrm{L} \\
\text { cerebellar }\end{array}$ & 15 & $\mathrm{~N}$ & 18 \\
\hline 16 & 56 & $\mathrm{~F}$ & 80 & Y & Lung & L parietal & 21 & $\mathrm{~N}$ & 18 \\
\hline 17 & 60 & $M$ & 80 & Y & Esophageal & $\mathrm{R}$ parietal & 8.9 & $\mathrm{~N}$ & 18 \\
\hline 18 & 56 & $\mathrm{~F}$ & 80 & Y & Undifferentiated & $\begin{array}{l}\mathrm{L} \\
\text { temporal }\end{array}$ & 3.1 & $\mathrm{~N}$ & 20 \\
\hline 19 & 69 & $M$ & 90 & $Y$ & Lung & L parietal & 4.3 & $\mathrm{~N}$ & 20 \\
\hline 20 & 69 & $M$ & 80 & $\mathrm{Y}$ & Lung & $\begin{array}{l}\text { L } \\
\text { occipital }\end{array}$ & 6 & $\mathrm{~N}$ & 18 \\
\hline
\end{tabular}


F: female. KPS: Karnofsky performance score prior to SLA. L: left. M: male. N: no. R: right. SCC: squamous cell carcinoma.WBRT: prior whole brain radiation therapy. Y: yes.

*Two lesions treated in the same patient.

Table 2. Stereotactic Laser Ablation and consolidation stereotactic radiosurgery_(SLA/CSRS).

\begin{tabular}{|c|c|c|c|c|c|}
\hline Patient & \%oflesion ablated & Days to cSRS & Laser system & cSRS & cSRS modality \\
\hline 1 & 77.4 & 30 & Monteris & 5 Gy x 5 & LINAC \\
\hline 2 & 74.6 & 23 & Monteris & 5 Gy $x 5$ & LINAC \\
\hline 3 & 98.2 & 23 & Monteris & 5 Gy x 5 & LINAC \\
\hline $4^{*}$ & 92.4 & 30 & Monteris & 5 Gy x 5 & LINAC \\
\hline $4^{*}$ & 100 & 37 & Monteris & 5 Gy x 5 & LINAC \\
\hline 5 & 100 & 23 & Monteris & 5 Gy x 5 & LINAC \\
\hline 6 & 100 & 26 & Visualase & 5 Gy x 5 & GK \\
\hline 7 & 73 & 16 & Visualase & 5 Gy x 5 & GK \\
\hline 8 & 100 & 19 & Visualase & 5 Gy x 5 & GK \\
\hline 9 & 97 & 30 & Visualase & 5 Gy x 5 & GK \\
\hline 10 & 100 & 40 & Visualase & 5 Gy x 5 & GK \\
\hline 11 & 100 & 27 & Visualase & 5 Gy x 5 & GK \\
\hline 12 & 100 & 35 & Monteris & 5 Gy x 5 & GK \\
\hline 13 & 92.4 & 27 & Monteris & 6 Gy x 5 & GK \\
\hline 14 & 97.8 & 24 & Monteris & 5 Gy x 5 & GK \\
\hline 15 & 99.2 & 20 & Monteris & 5 Gy x 5 & GK \\
\hline 16 & 100 & 25 & Monteris & 6 Gy x 5 & GK \\
\hline 17 & 99.3 & 20 & Monteris & 5 Gy x 5 & GK \\
\hline 18 & 100 & 73 & Monteris & 5 Gy x 5 & GK \\
\hline 19 & 90 & 24 & Monteris & 5 Gy x 5 & GK \\
\hline 20 & 98 & 36 & Monteris & 5 Gy x 5 & GK \\
\hline
\end{tabular}

CSRS: consolidation stereotactic radiosurgery (dose $x$ fractionation). Days to cSRS: days between stereotactic laser ablation and cSRS.Gy: Gray. GK: Gamma Knife. LINAC: linear accelerator. SLA: stereotactic laser 
ablation.\% lesion ablated: Percentage of contrast enhancing region of BMRS covered by blue contour line (Monteris) or orange pixel (Visualase).

*: two lesions treated in the same patient.

Table 3. Clinical outcomes

\begin{tabular}{|c|c|c|c|c|c|c|c|c|c|}
\hline Patient & $\begin{array}{l}\text { Post-SLA } \\
\text { deficit }\end{array}$ & $\begin{array}{l}\text { Post- } \\
\text { SLA } \\
\text { KPS }\end{array}$ & $\begin{array}{l}\text { Post- } \\
\text { CSRS } \\
\text { KPS }\end{array}$ & Readmission & LC & $\begin{array}{l}\text { Steroid } \\
(6 \mathrm{mo})\end{array}$ & $\begin{array}{l}\text { Steroid } \\
(12 \\
\mathrm{mo})\end{array}$ & $\begin{array}{l}\text { FLAIR } \\
(6 \\
\text { mo) }\end{array}$ & $\begin{array}{l}\text { FLAIR } \\
(12 \\
\text { mo) }\end{array}$ \\
\hline 1 & None & 90 & 90 & None & Yes & No & & No & \\
\hline 2 & None & 100 & 100 & None & Yes & No & & No & \\
\hline 3 & None & 90 & 90 & None & Yes & No & & No & \\
\hline $4^{*}$ & None & 80 & 80 & None & Yes & No & & No & \\
\hline $4^{*}$ & None & 80 & 80 & None & Yes & No & & No & \\
\hline 5 & None & 100 & 100 & None & Yes & No & & No & \\
\hline 6 & None & 100 & 100 & None & Yes & No & & No & \\
\hline 7 & None & 50 & 50 & Seizure & Yes & Yes** & & No & \\
\hline 8 & None & 100 & 100 & None & Yes & No & No & No & No \\
\hline 9 & None & 70 & 70 & None & Yes & No & & No & \\
\hline 10 & None & 90 & 90 & None & Yes & No & & No & \\
\hline 11 & None & 100 & 100 & $\mathrm{HA}$ & Yes & No & & No & \\
\hline 12 & None & 70 & 70 & None & Yes & Yes** & & No & \\
\hline 13 & None & 80 & 80 & None & No & Yes** & No & No & No \\
\hline 14 & None & 70 & 70 & None & No & No & No & No & No \\
\hline 15 & None & 80 & 80 & None & Yes & Yes** & & No & \\
\hline 16 & None & 60 & 60 & None & Yes & Yes** & & No & \\
\hline 17 & None & 80 & 80 & None & Yes & No & & No & \\
\hline 18 & None & 80 & 80 & None & Yes & No & No & No & No \\
\hline 19 & None & 90 & 90 & None & Yes & No & No & No & No \\
\hline 20 & None & 80 & 80 & None & Yes & No & & No & \\
\hline
\end{tabular}

cSRS: consolidation SRS. FLAIR: fluid attenuated inversion recovery.FLAIR ( $6 \mathrm{~m})$ : Expansion of FLAIR volume 6 months after cSRS (compared to FLAIR volume at the time of BMRS diagnosis). FLAIR (12 m): Expansion of 
FLAIR volume 12 months after cSRS (compared to FLAIR volume at the time of BMRS diagnosis).LC: local control. KPS: Karnofsky performance score. Mo: months.Post-SLA deficit: post-SLA neurological deficit. PostSLA KPS: KPS on day post-SLA treatment. Post-cSRS KPS: KPS one-month post-cSRS. Readmission: cause of 30-day readmission. SLA: stereotactic laser ablation. Steroid $(6 \mathrm{~m})$ : corticosteroid treatment 6 months after cSRS. Steroid (12 m): corticosteroid treatment 12 months after cSRS. SRS: stereotactic radiosurgery.

*: two lesions treated in the same patient.

**: corticosteroid was used to treat symptoms related to other brain metastases and unrelated to the SLA/cSRS treated BMRS.

Table 4. Outcomes of SLA and repeat radiosurgery monotherapy.

\begin{tabular}{|lll|}
\hline A. SLA & & \\
\hline Reference & Number of patients & Local control \\
\hline Ali et al. 2016 [13] & 23 & $61 \%$ at 4.6 months \\
\hline Ahluwalia et al. 2018 [14] & 20 & $74 \%$ at 3 months \\
\hline Bastos et al. 2020 [15] & 61 & $69.6 \%$ at 12 months \\
\hline
\end{tabular}

\begin{tabular}{|lllllll|}
\hline B. Repeat SRS & & & & & \\
\hline Reference & $\begin{array}{l}\text { \# of } \\
\text { patients }\end{array}$ & $\begin{array}{l}\text { \# of } \\
\text { BMRS }\end{array}$ & $\begin{array}{l}\text { Median SRS } \\
\text { dose (Gy) }\end{array}$ & $\begin{array}{l}\text { fractions of } \\
\text { SRS }\end{array}$ & LC $_{12}$ & $\begin{array}{l}\text { Adverse } \\
\text { radiation effect }\end{array}$ \\
\hline $\begin{array}{l}\text { Holt et al. 2015 } \\
{[30]}\end{array}$ & 15 & 15 & 21 & 3 & $75 \%$ & $13 \%$ \\
\hline $\begin{array}{l}\text { Minniti et al. 2016 } \\
{[10]}\end{array}$ & 43 & 43 & 21 & 3 & $70 \%$ & $19 \%$ \\
\hline $\begin{array}{l}\text { Rana et al. 2017 } \\
{[31]}\end{array}$ & 32 & 32 & 26.5 & 3 & $88 \%$ & $19 \%$ \\
\hline $\begin{array}{l}\text { Dincoglan et al. } \\
\text { 2019 [32] }\end{array}$ & 30 & 30 & 21 & 3 & 835 & $13 \%$ \\
\hline
\end{tabular}

A: Studies reporting stereotactic laser ablation as monotherapy for the treatment of brain metastasis that recurred locally afterradiosurgery (BMRS). B: Studies reporting repeat radiosurgery as monotherapy for the treatment of BMRS.SRS: stereotactic radiosurgery. LC $_{12}$ : local control at 12-months follow-up.

\section{Figures}




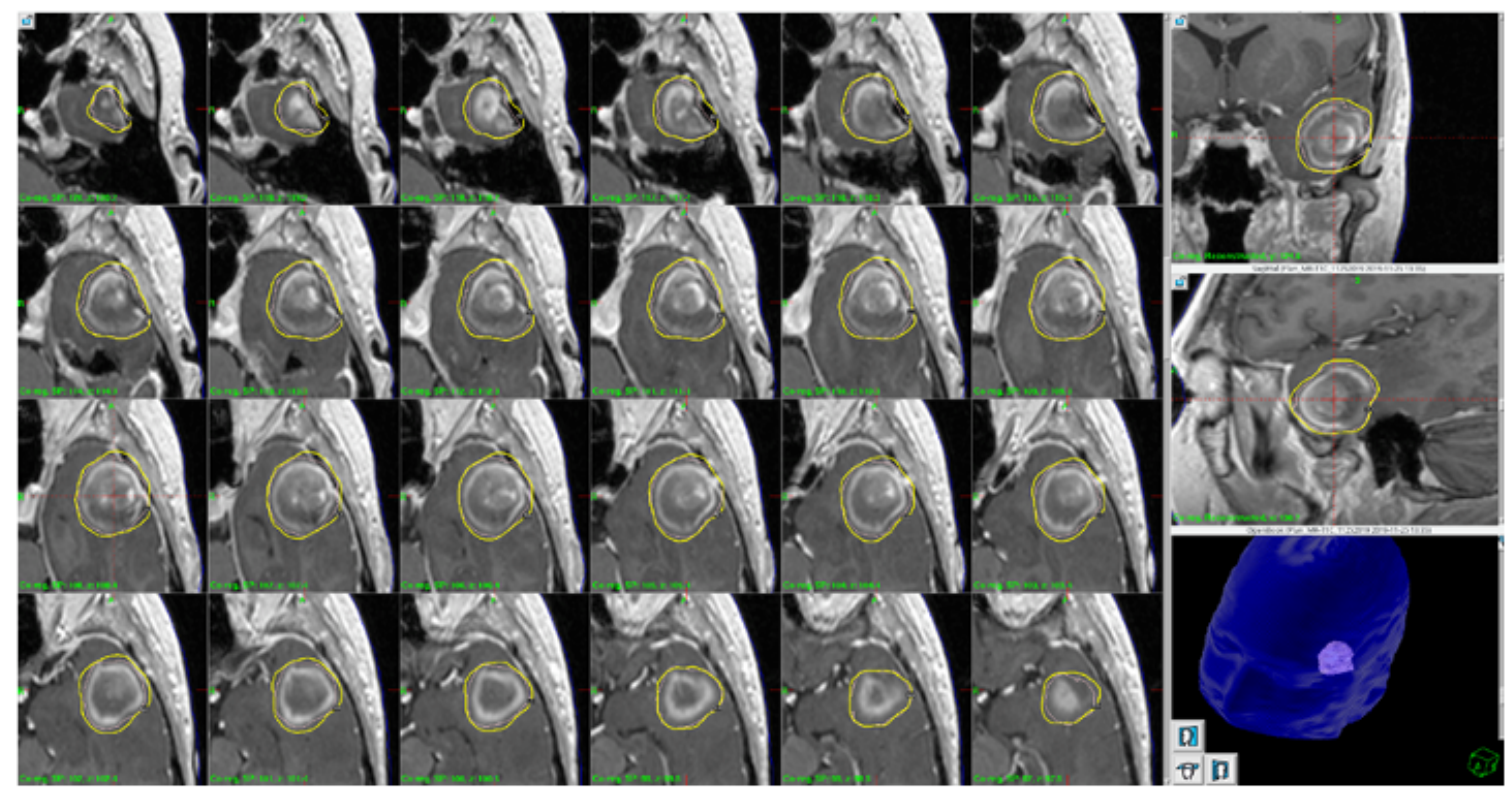

a

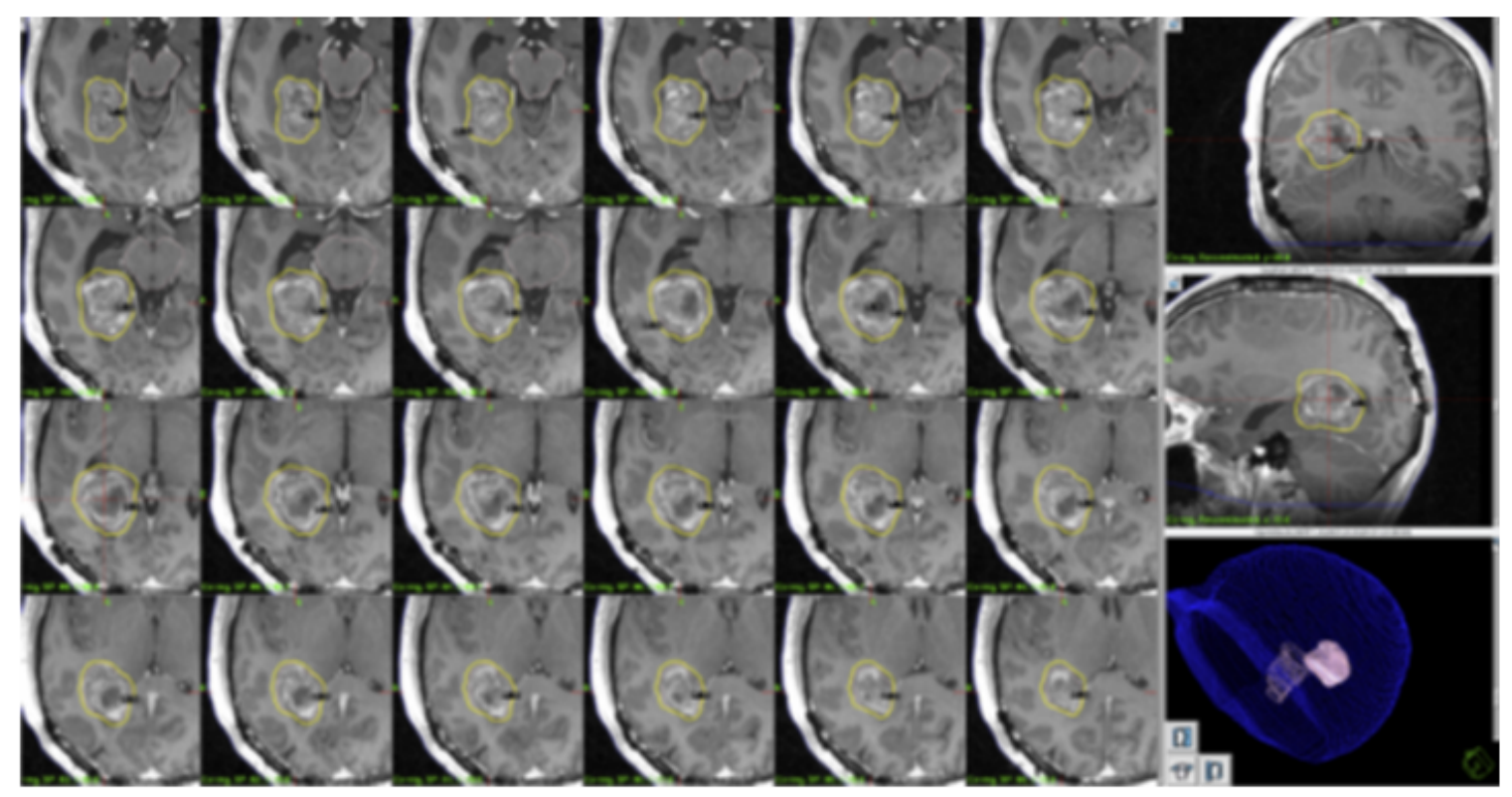

b

Figure 1

Representative consolidation stereotactic radiosurgery (cSRS) plans. Hypo-fractionated radiosurgery (5 Gy x 5) targeting the post-ablative region, including the rim of contrast enhancement was delivered. Radiosurgery plans for patient 8 (Figure 2A) and 9 (Figure 2B) are shown. 


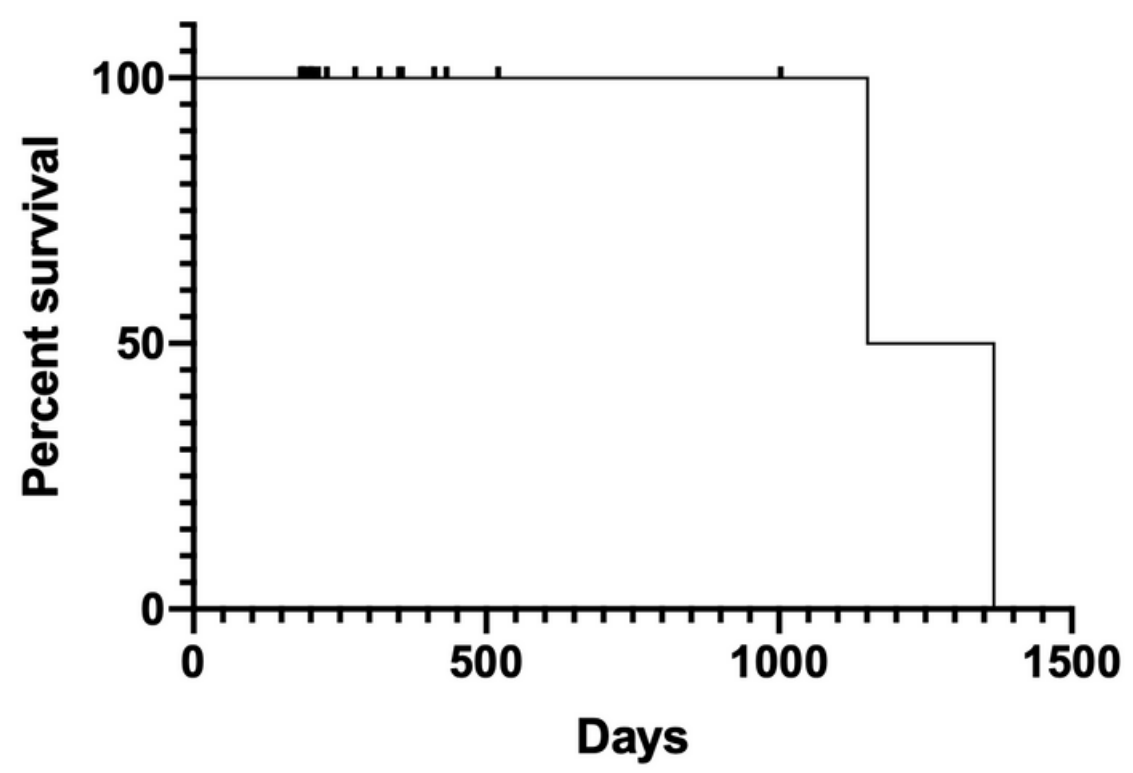

a

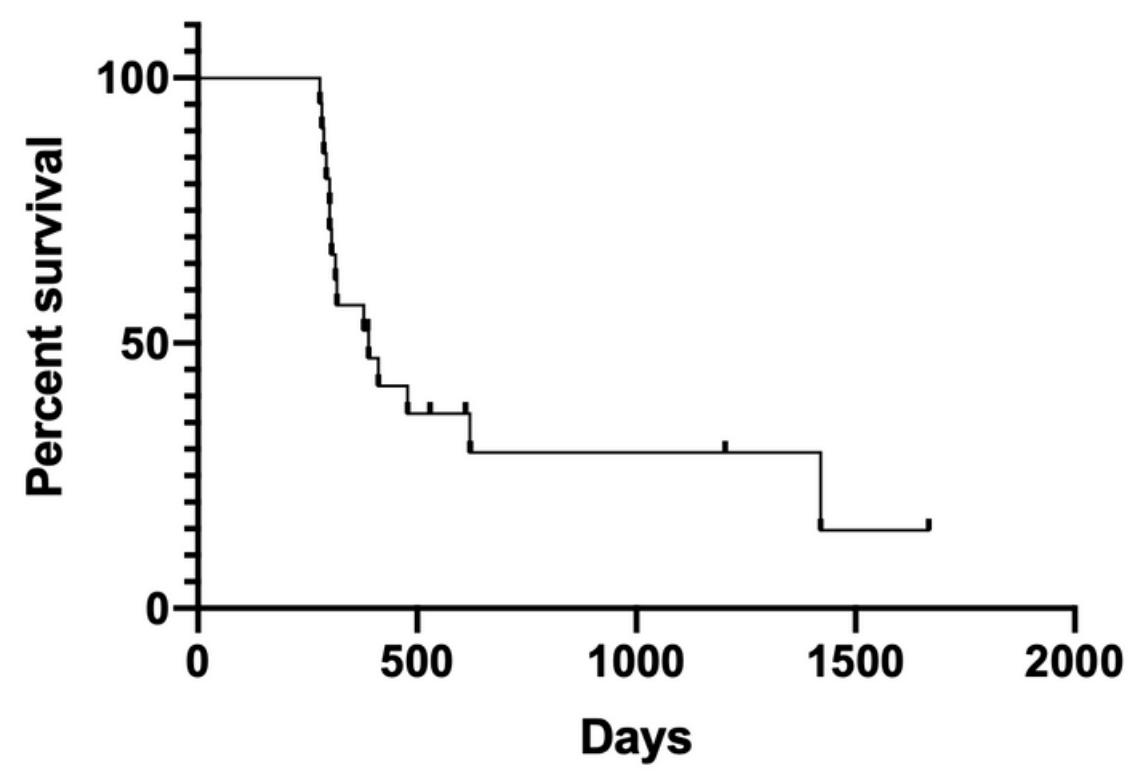

b

Figure 2

A: local control of brain metastasis that recurred locally after initial radiosurgery (BMRS) after Stereotactic Laser Ablation (SLA) followed by consolidation stereotactic radiosurgery (CSRS). B: overall survival of the study cohort. 


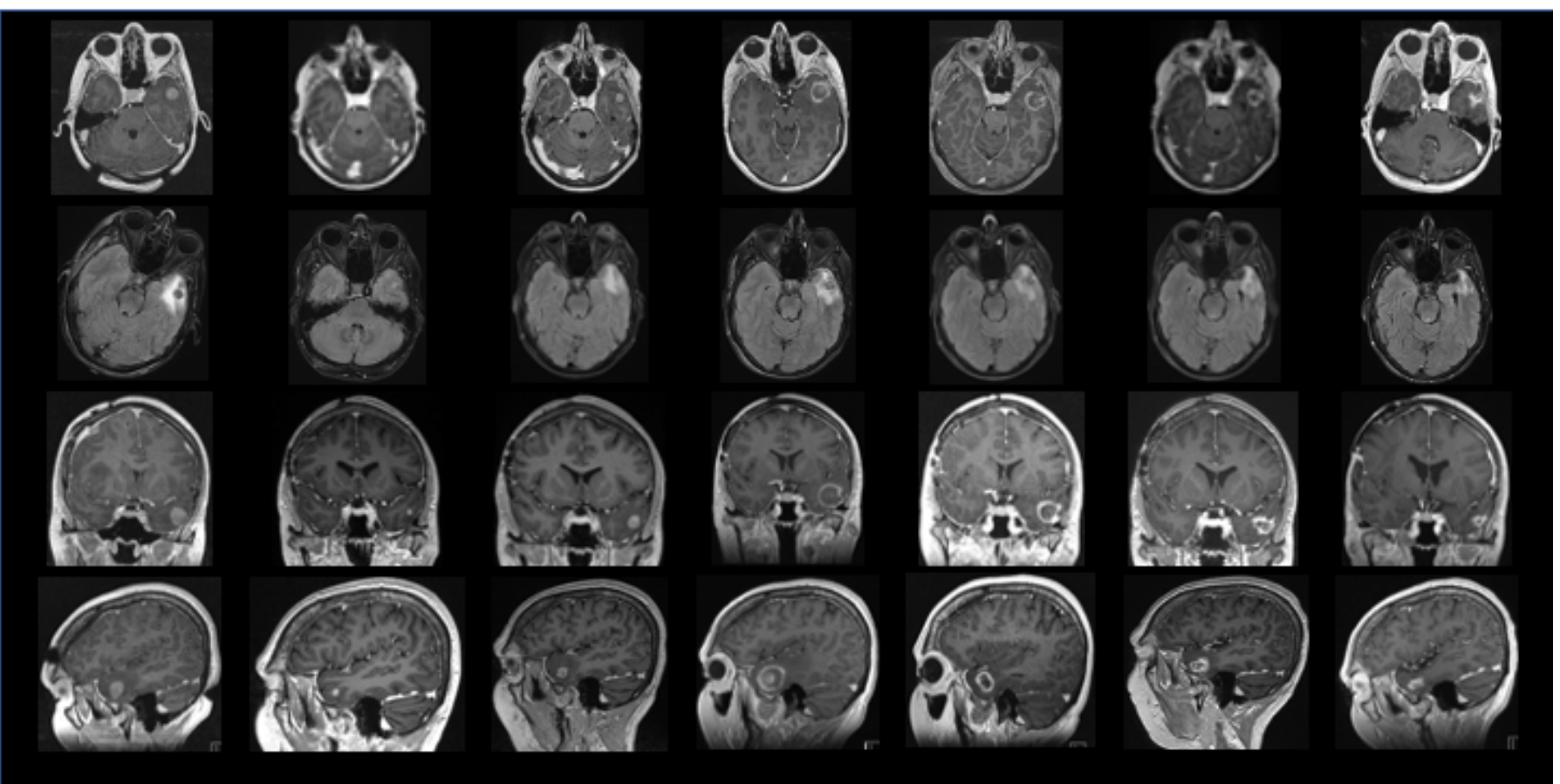

\section{Figure 3}

Sequential evolution of contrast enhancement and FLAIR volume (axial T1, axial FLAIR, coronal T1, sagittal T1, respectively from top to bottom) for representative SLA/cSRS treated lesion in patient 8 . This patient's BMRS remain controlled 19 months after SLA/cSRS. SRSi: initial SRS. BMRS was diagnosed one-year after the initial SRS. $3 \mathrm{~m} \mathrm{f} / \mathrm{u}$ : 3 months follow-up, $6 \mathrm{~m}$ f/u: 6 months follow-up, $19 \mathrm{~m}$ f/u: 19 months follow-up.
Pre-SRS1
$3 \mathrm{~m}$ post-SRS1
32 mo post-SRS1
SLA-cSRS
$3 \mathrm{~m} \mathrm{f} / \mathrm{u}$
$6 \mathrm{~m} \mathrm{f} / \mathrm{u}$
recurrence $(22 \mathrm{~m})$

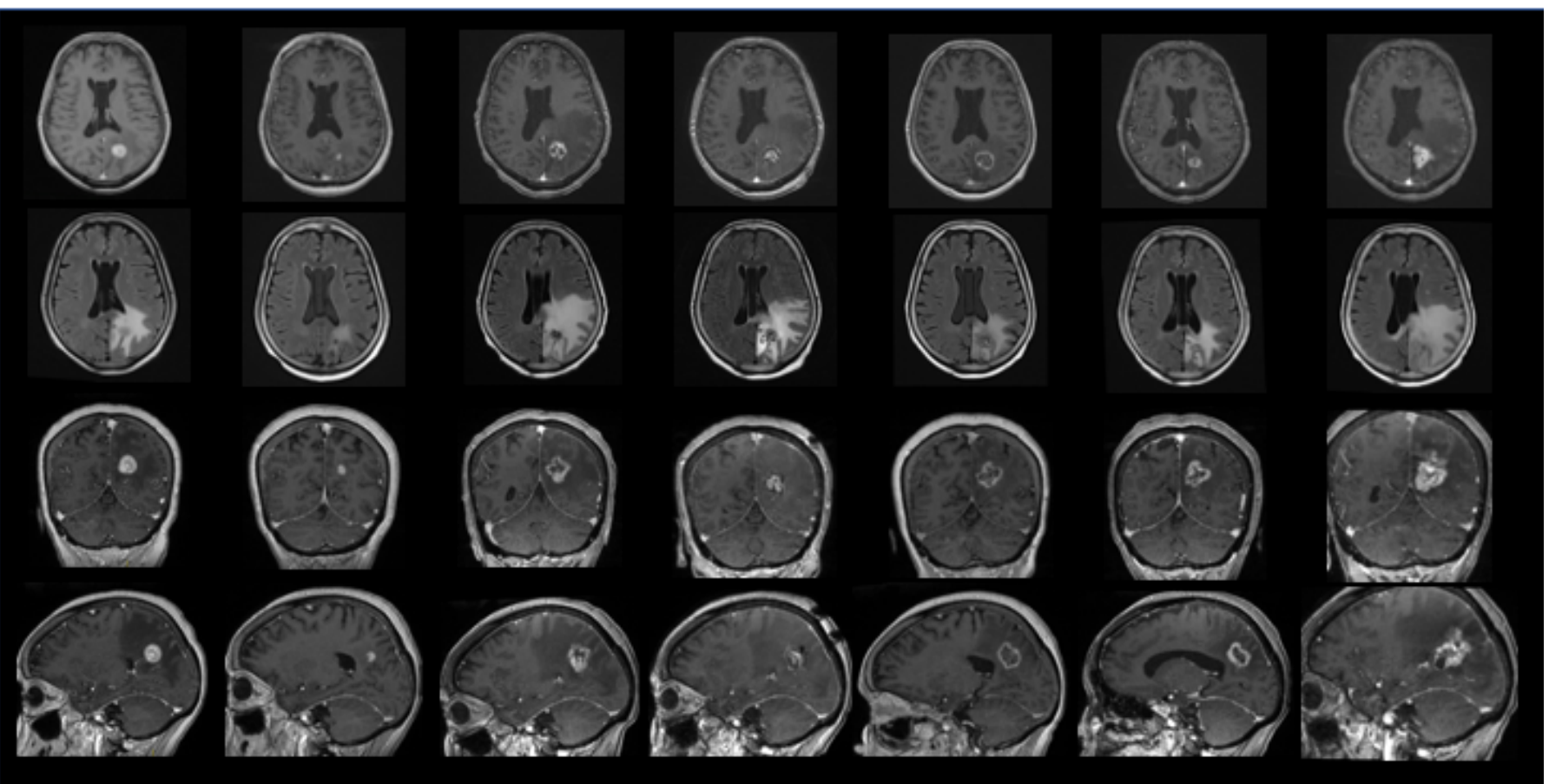

Figure 4 
Sequential evolution of local recurrence for patient 14. Contrast enhancement and FLAIR volume (axial T1, axial FLAIR, coronal T1, sagittal T1, respectively from top to bottom) demonstrate increase in contrast enhancement size and FLAIR volume at 22 months post SLA/cSRS. SRSi: initial SRS. BMRS was diagnosed 32 months after the initial SRS. $3 \mathrm{~m}$ f/u: 3 months follow-up, $6 \mathrm{~m}$ f/u: 6 months follow-up, recurrence $(22 \mathrm{~m})$ : tumor recurrence 22 months after SLA/cSRS. 